\title{
Performance Testing and Methodology for Evaluation of Power Line Communication
}

\author{
Petr Mlynek $^{1}$, Jiri Misurec ${ }^{1}$, Petr Toman ${ }^{1}$, Pavel Silhavy ${ }^{1}$, Radek Fujdiak ${ }^{1}$, Jan Slacik ${ }^{1}$, \\ Zeynep Hasirci², Konstantin Samouylov ${ }^{3}$ \\ ${ }^{1}$ Brno University of Technology, \\ Technicka 12, Brno, Czech Republic \\ ${ }^{2}$ Department of Electrical and Electronics Engineering, Karadeniz Technical University, \\ Turkey \\ ${ }^{3}$ RUDN University, \\ 6 Miklukho-Maklaya st, Moscow, 117198, Russia \\ mlynek@feec.vutbr.cz
}

\begin{abstract}
The paper describes a unified methodology which takes into account the advantages and disadvantages of different Power Line Communication (PLC) technologies suitable for Smart Metering deployment. The methodology should provide a detailed evaluation of PLC technologies based on a clear definition of the different parameters and their measurement. Based on this methodology, we should be able to provide a comparison of the selected technologies and the methodology should also provide data for the design of a telecommunication infrastructure for Smart Metering/Smart Grids. In this paper and based on this methodology, we also present the performance tests carried out in laboratory environment on low voltage and in on-field conditions on medium voltage.
\end{abstract}

Index Terms-Broadband communication; Power distribution lines; Smart grids; Simulation; Measurement.

\section{INTRODUCTION}

There is no doubt that the Smart Grid will exploit multiple types of communication technologies, ranging from fibre optics to wireless and to wireline. Therefore, the possibility of using the power grid and Power Line Communication (PLC) systems are a frequent topic of recent developments [1]-[3] because PLC uses existing wiring and no additional construction is necessary.

PLC is a technology that has matured to a level of high performance and worldwide deployment [4] but particular PLC technologies are not compatible and do not deliver the same level of performance for Smart Metering.

PLC technologies fall into three areas [1]:

- Ultra Narrow Band (UNB) operates at a very low data rate $(100 \mathrm{bps})$ in the low frequency band $(0.3 \mathrm{kHz}-$ $3 \mathrm{kHz}$ ). UNB uses one-way communication, used for load

Manuscript received 11 October, 2017; accepted 2 March, 2018

The research described in this paper was financed by the National Sustainability Program under grant LO1401 and by the Ministry of Interior of Czech Republic project No. VI20162018007.

The publication has been prepared with the support of the "RUDN University Program 5-100".

For the research, infrastructure of the SIX centre was used. control in particular. UNB has a very large operational range (hundreds of kilometres).

- Narrowband (NB) operates in a frequency band of $3 \mathrm{kHz}-500 \mathrm{kHz}(3 \mathrm{kHz}-148.5 \mathrm{kHz}$ in Europe). Singlecarrier NB technologies achieve data rates of a few kbps Low Data Rate NB-PLC (LDR NB-PLC). Nowadays, multicarrier technologies are capable of data rates of up to $800 \mathrm{kbps}$ - High Data Rate NB-PLC (HDR NB-PLC).

- Broadband (BB) operates in the high frequency band (1.8 $\mathrm{MHz}-250 \mathrm{MHz}$ ) and has data rates of several megabits per second up to hundreds of Mbps. This communication reaches lower communication distance (coverage) than narrowband and HDR NB-PLC ((hundreds of meters).

If we consider Smart Metering requirements alone [1], [5], a NB-PLC provides adequate data rates. On the contrary, BB-PLC provides solution for broad-bandwidth requirements to fulfil the evolution of Smart Grid applications but an estimation of why much higher data rates may be needed is still missing. Therefore, this article focuses on the communication potential of BB-PLC technology.

PLC is well suited for quick and inexpensive deployments for Smart Metering/Smart Grids, but the grid in not the same in any utility, because of particular grid evolution, architecture, circumstances, material etc. Therefore, the contribution of the article is to show by methodical experimentation that BB-PLC performance in the presence of similar grid conditions but in a different location is varied.

We also exploit in this article our novel idea for simple guidelines and repeatable methodology for evaluation and performance testing of BB-PLC technology.

The article is divided as follows: In the first part, we propose the reproducible and repeatable methodology and introduce a test bed. In the second part, the results of measurements and performance tests for the laboratory environment and the real field test are introduced. Finally, the comparison of different technologies was carry out and summary and discussion are presented. 


\section{A. Evaluation and Performance of PLC Technologies - State of the Art}

PLC network performance is usually described using the phrase "up to". This is not an absolute rating, because in a real PLC network not all point-to-point connections are the same. The amount of signal attenuation and noise between outlets is not the same. These differences require that PLC technologies be tested and performance-compared in the presence of typical noise sources.

In the vast majority of the literature various data rate limits and data rate measurements or simulation results are introduced. Data rates are sometimes chaotically presented on the physical layer together with data rates on the application layer. The application data rate is significantly lower than the physical data rate. The application data rate measurements are missing.

Most of the broadband power line communication (BBPLC) standards adopted the OFDM modulation technology [1], [6]-[8], for examples HomePlug AV/Extended, HomePlug Green PHY, HD-PLC, UPA Powermax, Gigle MediaXtreme, IEEE 1901 and ITU-T G.hn (G.9960/G.9961).

The HDR NB-PLC standards also adopted the OFDM modulation technology [9]. Therefore, the performance evaluation of the OFDM system in the power line environment is essential. The article presents mainly a methodology for the performance evaluation of the OFDM system in the power line environment.

Many results of performance evaluation or tests of PLC on low voltage (LV) lines have been reported in the last decade, we point out the most prominent ones: [6], [5], [10][19]. These publications mainly focus on modelling and simulation. Many of them also focus on PLC in the $0.2 \mathrm{MHz}-30 \mathrm{MHz}$ range, so they are more applicable for inhome PLC networks. Only a few of these publications [6], [20] were presented a performance evaluation methodology for PLC. In [6], [11], the methodology was adopted via simulations. In [5], [20], the parameters for PLC-based Smart Metering assessment (especially for availability and stability) were introduced. Therefore, this article focuses on performance tests and real measurements based on our proposed methodology.

The methodology for the performance of OFDM systems has been an active area of wireless communication research, e.g. in the articles [21]-[25]. But in PLC systems, particular factors have to be considered, e.g. noise, cable type, loading, impedances and time-frequency selective channel. An important feature relevant to all types of noise is their daytime and frequency dependency.

Many studies of PLC on medium voltage (MV) and high voltage (HV) lines have been presented in the last decade [26]-[36]. These publications focus mainly on the simulations of the behaviour of PLC system in MV and HV, high frequency behaviour of $\mathrm{HV}$ and MV power lines, simulations of signal propagation through MV power transformers, MV cable model and experimental validation in laboratory.

Only in [35], were the on-field experimental tests of narrowband power line communication between two secondary substations connected by an MV cable power line carried out.

Real on-field experimental measurements of BB-PLC are missing and on-field experimental tests of achievable data rates of BB-PLC on MV are also missing. The article [36] focuses on achievable data rates of BB-PLC on MV, but only via simulation and only for underground lines.

Only the articles [36] and [37] focus on performance evaluation of PLC with real field testing, but with sharp focus only on throughput, latency and packet loss for specific scenarios. In the present article we want to propose a reproducible and repeatable methodology.

Therefore, the article also focuses on on-field experimental tests of BB-PLC and especially on achievable data rates of BB-PLC in overhead transmission MV lines.

The contribution of this article is three-fold: First, a reproducible and repeatable methodology for the evaluation of PLC technologies for Smart Metering deployment is proposed. Second, experimental measurements based on the proposed methodology for a laboratory environment and also for an on-field scenario are conducted. Third, a particular BB-PLC system is evaluated via real measurements, because these BB-PLC systems have a drawback in that they lack proven BB-PLC technologies.

\section{Methodology for Evaluation OF Power Line COMMUNICATION}

Measurements and performance tests in laboratory environments and especially in on-field are the key to developing and optimizing PLC communication for Smart Metering deployment. Measurements and performance tests for all PLC technologies perform best when very simple guidelines are followed. These guidelines should be considered (best practices) for any PLC evaluations and measurements and will help ensure best results and future comparison.

The key parameters for the evaluation of Smart Metering network behaviour and performance are the availability and stability [38].

The key parameters for the evaluation of the communication performance of a Smart Metering network are the data rate (throughput), latency, robustness, noise immunity, effective communication distances and adaptation of the communication paths to network topology changes.

Therefore, the focus in the performance tests and methodology in this article is on the following parameters (guidelines):

1. Throughput, latency and losses for the UDP protocol according to RFC 2544. The impact of the different distributions of frame, broadband and narrowband noise on throughput.

2. Throughput for the TCP protocol according to RFC 6349. The impact of broadband and narrowband noise on TCP throughput.

3. Throughput on the physical layer (PHY data rate) and application throughput.

4. TCP and UDP throughput comparison.

The frame size may have a significant impact on the communication performance. Therefore, an exact frame size 
should be specified for all these measurements. The frame size is chosen to cover the most important frame sizes which might be present in the network. The distribution for RFC 2544 looks like this: 64 bytes, 128 bytes, 256 bytes, 512 bytes, 1024 bytes, 1280 bytes and 1518 bytes.

\section{MEASUREMENT SETUP}

Testing PLC devices in the laboratory allows a test methodology to be employed that is reproducible and repeatable. Figure 1 illustrates a test bed with a separated and isolated power network between two powerline communication devices. The test bed also allows various impairment devices to be added to the test environment in a manner that allows tests to be performed in a controlled and reproducible manner.

PLC devices work best when they have direct and separate access to the AC line. Therefore, we used EMI filtering, which works to further isolate equipment noise from the line.

The test bed includes:

- Headend (HE) and Repeater (REP) of Corinex Low

Voltage and High Density Compact Gateway.

- NetBlazer measurement tool.

- Noise generator PROPOWER - 1 .

- Generator Agilent 33521A and coupling unit.

- Signal-to-Noise Ratio (SNR) tool.

- Line filtering for isolation.

The LV Compact Gateway [39] and the HD Compact Gateway [40] are Corinex BB-PLC access product line using the AV200 Technology. The AV200 Powerline technology by Corinex provides physical layer transfer rates of up to 200 Mbps based on the OFDM technology. For measurements, modems without Power Mask in the 2.9 MHz-12.9 MHz frequency band are used.

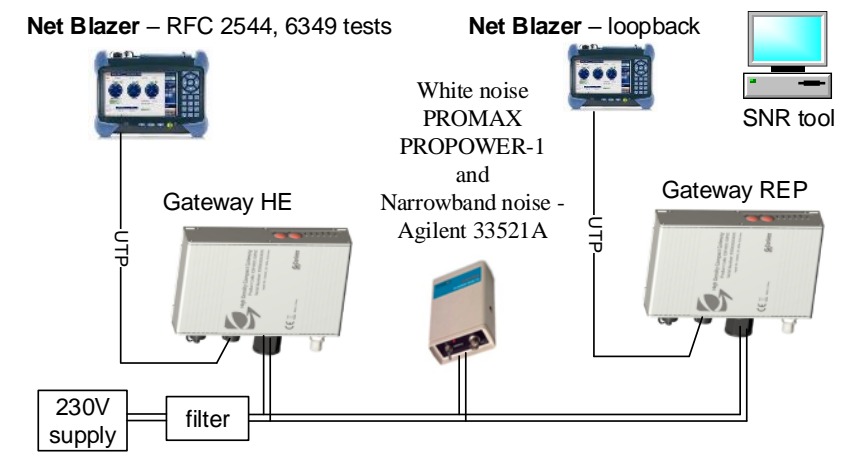

Fig. 1. Measurements set-up.

The following measurement scenarios were considered for parameters (guidelines) from A) to D) with an average Signal-to-Noise Ratio (SNR):

1. Modems connected via coaxial cable (SNR $36.5 \mathrm{~dB}$ ).

2. Modems connected via direct LV line of $50 \mathrm{~m}$ in length (SNR $25.1 \mathrm{~dB})$.

3. Modems connected via direct $\mathrm{LV}$ line of $50 \mathrm{~m}$ in length and with $\mathrm{NN}$ on $7 \mathrm{MHz}$ (bandwidth $600 \mathrm{kHz}$ ) (SNR $21.3 \mathrm{~dB})$.

4. Modems connected via direct LV line of $50 \mathrm{~m}$ in length and with broadband noise (SNR $16.7 \mathrm{~dB}$ ).
5. Modems connected via direct LV line of $50 \mathrm{~m}$ in length and with noise in the form of second HeadEnd (master) (SNR 10.6 dB).

Figure 2 shows the SNR for particular scenarios. This figure shows the impact of a particular noise on SNR. Interfering sources generate noise and attenuate the signal. This interference leads to a lower SNR and a lower throughput data rate.

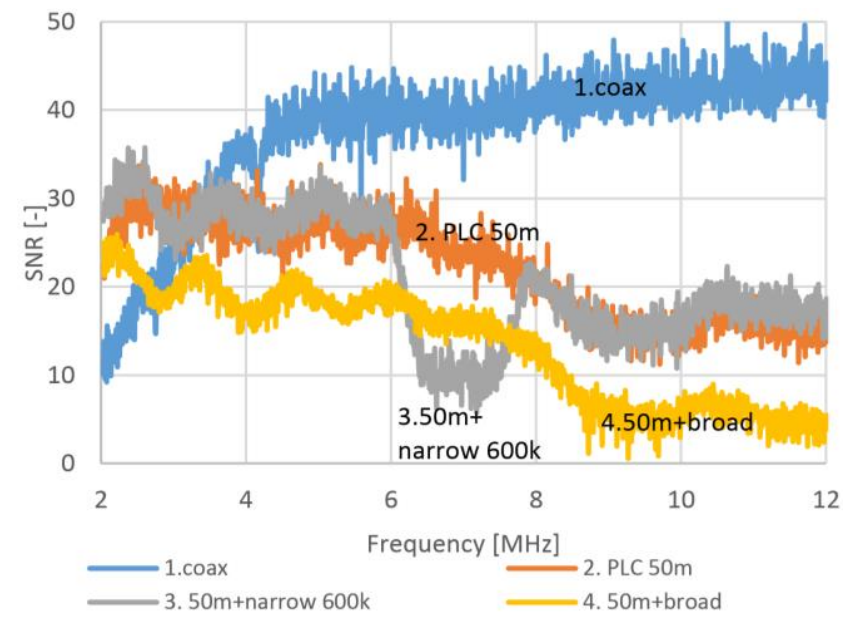

Fig. 2. Comparison of SNR for a particular scenario.

The narrowband noise on the $7 \mathrm{MHz}$ frequency was chosen as an example. The narrowband noise caused a decrease of $4 \mathrm{~dB}-6 \mathrm{~dB}$ in the average SNR. This decrease caused only a small decrease in the total throughput.

The broadband noise provides a SNR decrease of $8.4 \mathrm{~dB}$ while noise in the form of second HeadEnd (master) provides a SNR decrease of $16 \mathrm{~dB}$. The broadband noise caused a decrease in the SNR over the whole frequency range and thereby the data rate decreased significantly.

\section{Performance Tests And Measurement Results in LABORATORY ENVIRONMENT}

\section{A. Throughput for UDP According to RFC 2544}

Figure 3 and Fig. 4 show the UDP throughput on the network L3 layer for symmetrical data flow (data flows in both directions upstream and downstream). In these figures, we can see that the different frame sizes had a significant impact on the throughput. Figure 4 shows the general impact of the frame size on throughput for overall scenarios. Throughput for a frame size of $64 \mathrm{~B}$ is two or three times lower than throughput for the frame size of $1518 \mathrm{~B}$. For an evaluation of the transmission capacity of the PLC network for the UDP protocol, measurements with different frame size are essential, as well as the knowledge of the size of the transmitted frames of the target application.

The impact of broadband noise on the throughput is also shown in Fig. 3. The best condition for transmitting was achieved using a coaxial cable connection between modems, where the maximum data rate was $28.7 \mathrm{Mbps}$ for a frame of $1528 \mathrm{~B}$ in size. Under the same conditions, the physical data rate was $44 \mathrm{Mbps}$.

In the case of narrowband noise (scenario no. 3) and in comparison with the best PLC conditions (scenario no. 2), 
the SNR was decreased by a narrowband noise of $4 \mathrm{~dB}-6 \mathrm{~dB}$ and the data rate was decreased by a narrowband noise of 8 Mbps (Fig. 3).

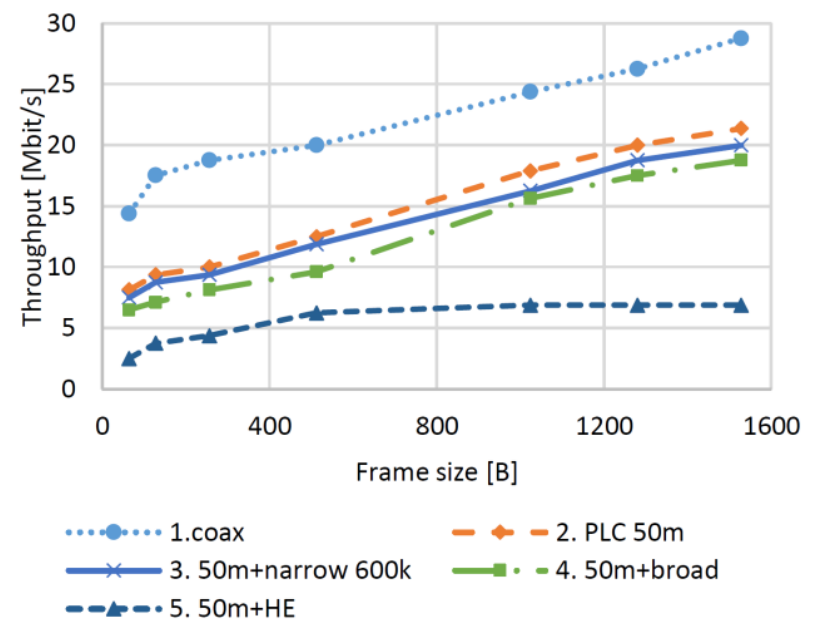

Fig. 3. Impact of frame size on throughput for different scenarios.

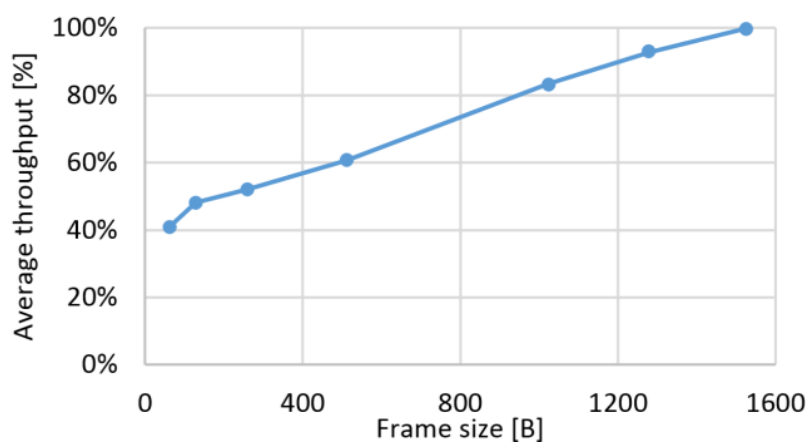

Fig. 4. General impact of frame size on throughput for overall scenarios.

The most powerful noise was obtained in the connection of second HeadEnd master modem (scenario no. 5). This modem used a different power mask, which avoided some frequency bands. These omitted frequency bands were used for the transmission of the first HeadEnd modem with throughput of $7 \mathrm{Mbps}$.

Figure 5 shows the impact of frame size on frame loss for a particular scenario. The impact of frame loss in this figure is given by the way of testing according to RFC 2544, when the tester sends data to the modem with successively increasing transmission rate in steps of $10 \%$. If this rate is higher than the rate of linked connection between PLC modems, there is a loss of frames.

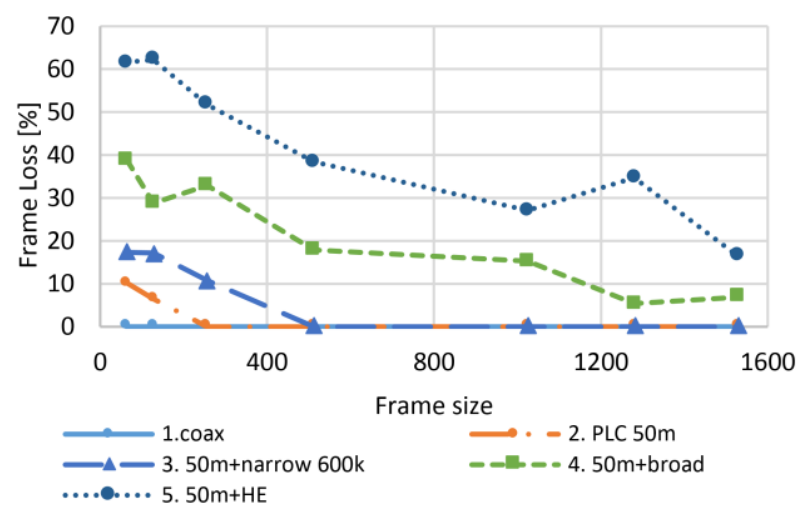

Fig. 5. Impact of frame size on frame loss for different scenarios.

The limit of BB-PLC technology was reached with an increase in latency (Fig. 6) and frame loss for scenarios No. 4 and No. 5 with broadband noise.

According to these results, we can evaluate the communication performance in terms of:

- FER $<10 \%$, latency $<800 \mathrm{~ms}$ and throughput over $5 \mathrm{Mbps}$ for SNR $>20 \mathrm{~dB}$ and minimum frame size $512 \mathrm{~B}$, - FER $<0.1 \%$, latency $<400 \mathrm{~ms}$ and throughput over $10 \mathrm{Mbps}$ for SNR > $16 \mathrm{~dB}$ and frame size $1024 \mathrm{~B}$.

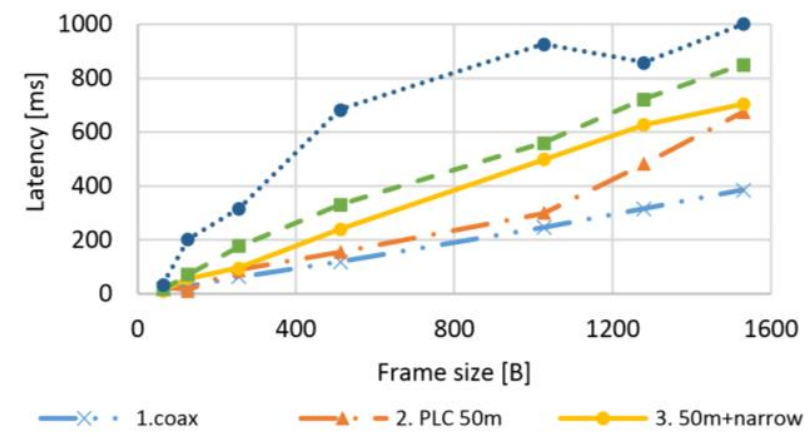

$-=-4.50 \mathrm{~m}+$ broad $\cdots \cdot \cdots \cdot .5 .50 \mathrm{~m}+\mathrm{HE}$

Fig. 6. Impact of frame size on latency for different scenarios.

\section{B. Throughput for TCP Protocol According to RFC 6349}

Figure 7 shows the TCP throughput on the transport L4 layer for asymetrical data flow (data flow only in one direction upstream or downstream) and TCP window size of $33 \mathrm{KiB}$.

TCP, unlike the UDP protocol, provides a reliable acknowledged transmission (retransmission of loss frames) but it is less efficient (lower throughput). UDP sends as fast as you can and TCP connection is first established.

The RFC 6349 methodology is composed of the following phases:

- The maximum transmission unit (MTU) of the line detection.

- RTT measurements and calculation of the optimal window for the TCP protocol.

- TCP throughput testing, TCP efficiency (how many bytes were re-sent) testing, and delay of buffer (how many times the RTT increased) testting.

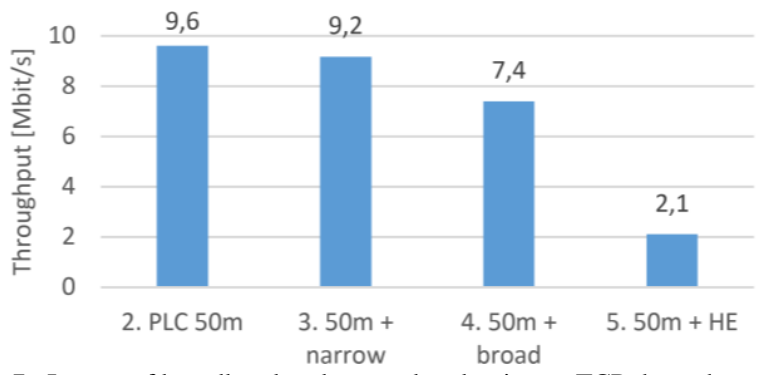

Fig. 7. Impact of broadband and narrowband noise on TCP throughput.

\section{Throughput on the Physical Layer (PHY Throughput)}

Table I shows a comparison of throughput on the application layer and throughput on the physical layer. Throughput on the application layer was measured by the NetBlazer and according to the RFC 2544. Table I shows UDP throughput for the network L3 layer and symmetrical data flow for scenario no. 2 (ideal power line condition). This throughput increases with increasing size of frame. Throughput on the physical layer was measured using the 
Corinex modem. From this table, we can see the significant differences between the PHY throughput and the network L3 layer data throughput (according to the RFC 2544). This significant difference holds especially for BB-PLC systems with the throughputs of tens to hundreds of Mbps, where there is a significant predisposition to errors and therefore it is necessary to allocate part of the transmission capacity for error detection and correction.

TABLE I. THROUGHPUT COMPARISON.

\begin{tabular}{|c|c|c|c|}
\hline \multicolumn{2}{|c|}{ Application layer throughput } & \multicolumn{2}{|c|}{ Physical layer throughput } \\
\hline \multirow{2}{*}{$\begin{array}{c}\text { Frame size } \\
{[\mathrm{B}]}\end{array}$} & \multirow{2}{*}{$\begin{array}{c}\text { Throughput } \\
\text { [Mbps] }\end{array}$} & $\mathbf{T X}$ & $\mathbf{R X}$ \\
\hline & & \multicolumn{2}{|c|}{ XPUT [Mbps] } \\
\hline 64 & 7.50 & \multirow{5}{*}{34} & \multirow{5}{*}{24} \\
\hline 256 & 8.13 & & \\
\hline 512 & 9.63 & & \\
\hline 1024 & 16.25 & & \\
\hline 1528 & 21.38 & & \\
\hline
\end{tabular}

\section{TCP and UDP Throughput Comparison}

As mentioned in section B, the UDP throughput is in general higher than the TCP throughput. Therefore, the goal of measuring was to compare the differences between UDP and TCP throughputs for PLC modems. These measurements were carried out for the freqeuncy band 2.9 MHz-32 MHz (Mode 6 according to the vendor).

According to the vendor, the physical throughput is $200 \mathrm{Mbps}$, but the modes established connection with $100 \mathrm{Mbps}$ interface. The modems also dynamically allocated the bandwidth in the upstream and downstream data flows.

Figure 8 shows the impact of the different shapes of generated UDP data flows on UDP throughput (UDP throughput for the small frame size $64 \mathrm{~B}$ ). The initial shape of generated UDP data flows is the shape for $100 \mathrm{Mbps}$ and then it is partially reduced to $50 \%$ and $25 \%$.

Figure 8 also shows the influence of distance on throughut for different UDP traffic streams (UDP traffic streams are generated with $25 \mathrm{Mbps}, 50 \mathrm{Mbps}$ and $100 \mathrm{Mbps}$ ). It is obvious that the throughput is significantly reduced for higher generating speeds of UDP traffic streams (the modem has become overloaded). For example, in the case of UDP traffic stream generation of $100 \mathrm{Mbps}$, the throughput drops to $3 \mathrm{Mbps}$ versus $18 \mathrm{Mbps}$ for UDP traffic generation of $25 \mathrm{Mbps}$.

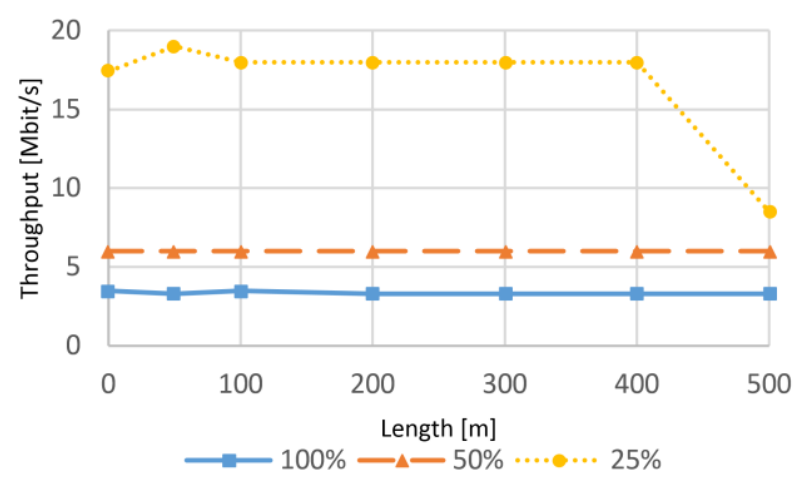

Fig. 8. Influence of distance on throughut for different UDP traffic streams (UDP traffic streams are generated with $25 \mathrm{Mbps}$, $50 \mathrm{Mbps}$ and $100 \mathrm{Mbps}$ ).

Figure 9 shows a comparison of UDP throughput for different frame sizes and shapes of generated UDP data flows and also TCP throughput for a pair of streams. It can be seen that a full vendor throughput of $100 \mathrm{Mbps}$ can be achieved in the case of unidirectional UDP data flow with large frame sizes, e.g. 1500 B. The UDP throughput significantly drops for smaller frame sizes.

The TCP throughput for a TCP window size of $16 \mathrm{KiB}$ was $38 \mathrm{Mbps}$ and the TCP throughput decreased with distance (Fig. 9, red waveform). The significanty lower throughput for TCP is caused by the dynamic allocation of bandwidth in the upstream and downstream data flows.

Therefore, the throughput in different scenarios or power grid conditions is hard to estimate from the vendor physical throughput. The throughput in ideal power line conditions depends on the size of the frames as well as on the UDP/TCP protocol type.

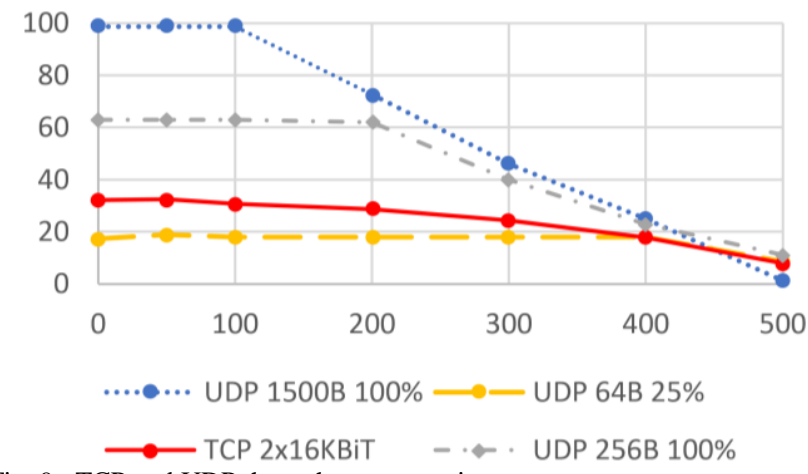

Fig. 9. TCP and UDP throughput comparison.

\section{PERformance Tests AND MEASUREMENT Results in REAL FIELD - MEDIUM VOLTAGE LINES}

Thanks to the cooperation with a local power distribution company E.ON, the real medium voltage (MV) grid scenario was used for measurements based on the proposed methodology.

Figure 10 shows a MV line configuration with three MV nodes and distances between the nodes. Modem no. 1 is Headend (master modem). Modems no. 2 and 3 are the repeaters. Modem no. 3 is located in the section switch (circuit breaker). The $13.4 \mathrm{MHz}-23.4 \mathrm{MHz}$ frequency band was used. Steel-reinforced aluminum-conductor cable was used in overhead transmission lines of $22 \mathrm{kV}$. Capacitive couplers were used for broadband signal injected into the power lines. The OVERCAP capacitive coupling solution was considered (phase-to-ground, two phase). The distance of 906 meter was the longest MV line without repeaters.

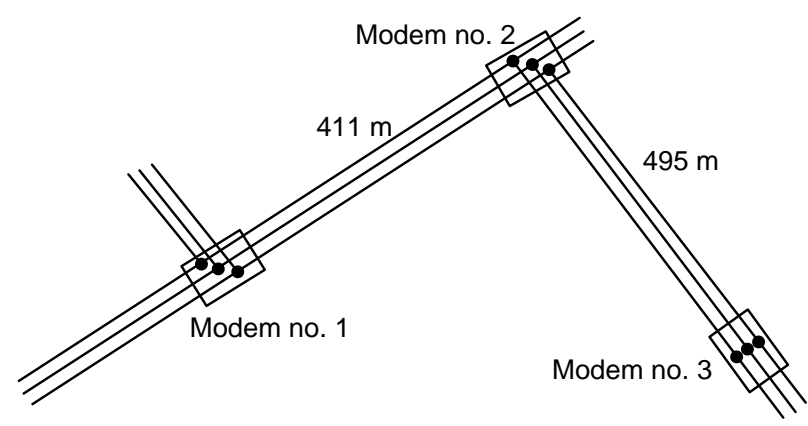

Fig. 10. MV real field grid scenario.

Figure 11 shows a UDP throughput according to the RFC 2544 for different distributions of frame sizes and two distances (without repeater). The maximum communication 
distance without repeaters, which could be measured was 906 meters and the throughput for this distance was approximately $11.75 \mathrm{Mbps}$ for $1280 \mathrm{~B}$ frame size of. The impact of one repeater is not so significant: the outage of one repeater or a reduction of the number of repeaters (optimization) will lead to a lower throughput but communication will still be possible.

The TCP throughput according to the RFC 6349 was 7.2 Mbps for 411 meters and 1.8 Mbps for 906 meters.

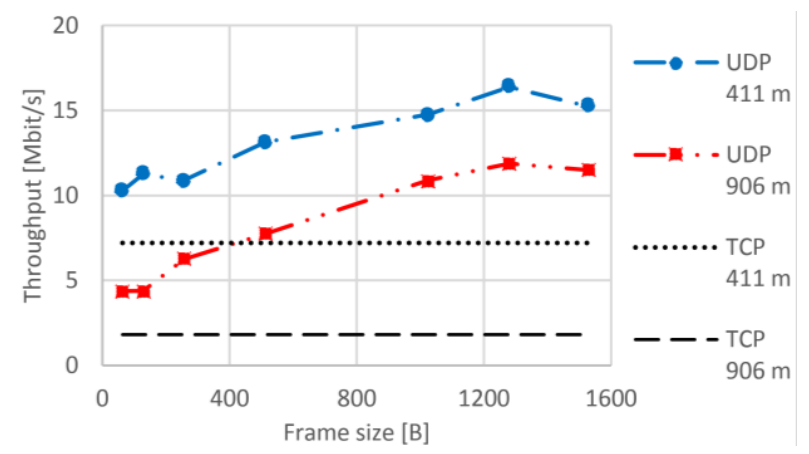

Fig. 11. UDP and TCP throughput on MV lines.

Figure 12 shows the channel frequency response (CFR) for a MV line between modems No. 1 and No. 3. The waveform of CFR is very wavy (many notches) in comparison with LV lines [42]-[45], but the notches are not deep. Shallow spectral notches are observed, therefore the whole frequency band can be used for transmission.

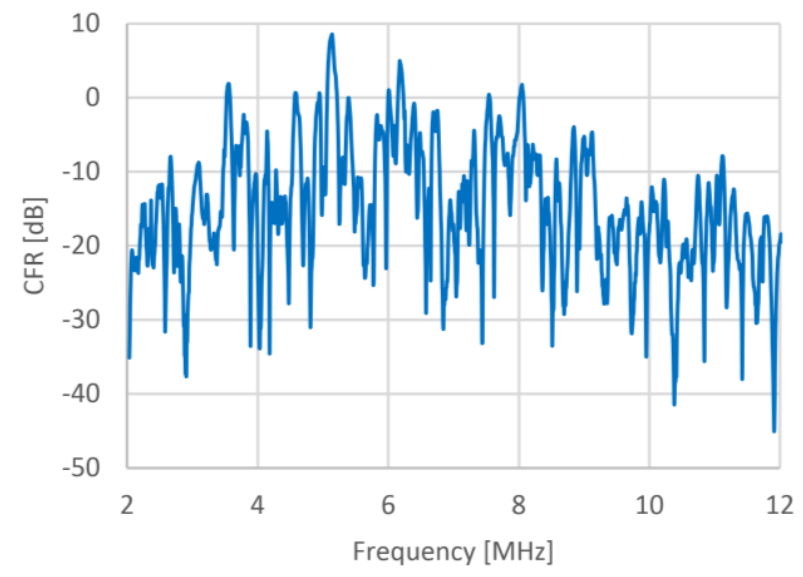

Fig. 12. CFR for MV line (modem no. 1-no. 3).

\section{COMPARISON OF DIFFERENT PLC TECHNOLOGIES}

Based on propoused methodology, we are able to provide a comparison of the selected PLC technologies. Figure 13 shows a comparison of the throughput for Corinex and HomePlugAV2 (Linksys) modems. The laboratory test bench (scenario no. 2) with modems connected via direct LV line of $50 \mathrm{~m}$ in length was considered. The average SNR of this scenario was $25.1 \mathrm{~dB}$. From this result, it is clear that Linksys achieves higher throughputs. But we have to consider that these broadband modems use a different frequency bands. HomePlug AV2 operates in $2 \mathrm{MHz}-$ $86 \mathrm{MHz}$ and CORINEX in 2.9 MHz-12.9 MHz.

On the other hand, the channel capacity $C$ is given by

$$
C=B \times \log _{2}(1+S N R)
$$

where $B$ is channel bandwidth and SNR is Signal-to-Noise Ratio for particular power line channel. The throughput of a particular system $R$ is given by

$$
R=k \times B \times \log _{2}(1+S N R),
$$

where $k<1$ represents the optimal usage of channel capacity $C$ and overhead of all layers of the TCP/IP (OSI) model. For example, if we consider the AWGN channel, CENELEC limits of $134 \mathrm{~dB} \mu \mathrm{V}$ over the signal bandwidth and signal power density of $120 \mathrm{~dB} \mu \mathrm{V} / 200 \mathrm{~Hz}$, the difference in the SNR of two systems using different bandwidths and overheads is given by

$$
\Delta S N R_{d B}=10 \times \log _{10}\left(\frac{2^{\frac{R_{1}}{k_{1} \times B_{1}}}-1}{2^{\frac{R_{2}}{k_{2} \times B_{2}}}-1}\right) .
$$

According to the throughput $R$ in Fig. 13 and the SNR for the scenario No. 1) $(25.1 \mathrm{~dB})$ the coefficient is $k=0.287$. Therefore, the comparison of SNR for AV2 and Corinex in the case of the simplification $k \approx k_{1} \approx k_{2}$ is given by

$$
\Delta S N R_{d B}=10 \times \log _{10}\left(\frac{2^{\frac{21 \times 10^{6}}{0.287 \times 10 \times 10^{6}}}-1}{2^{\frac{48 \times 10^{6}}{0.287 \times 84 \times 10^{6}}}-1}\right)=17.3 \mathrm{~dB}
$$

From this result, it is obvious that the SNR for Corinex is higher by about $17.3 \mathrm{~dB}$. Nevertheless the throughput is two times lower. This result highlights that it is not possible to compare the SNR of different systems using different bands because the SNR is calculated as

$$
S N R=\int \frac{P S D_{-} r e c(f)}{S U M(f)} d f,
$$

where PSD_rec is Power Spectral Density (PSD) of the received signal. The channel bandwidth $B$ is the difference between Corinex and AV2. The theoretical bandwidth comparison $\Delta B$ is given by

$$
\Delta B=\frac{B_{A V 2}}{B_{C O R}}=8.4 .
$$

The bandwidth ratio from (6) will be significantly lower due to the higher attenuation on higher frequencies, lower power on higher frequencies and different PSD.

According to the lower average SNR for AV2 than for Corinex from (4) and the higher bandwidth for AV2 than for Corinex from (6) the different systems could be compared, but Fig. 13 shows that the actual throughput of AV2 is only 2.5 times higher than that of Corinex.

The broadband frequency range of the HomePlug AV2 will also achieve shorten communication distances and noise immunity will be lower. 


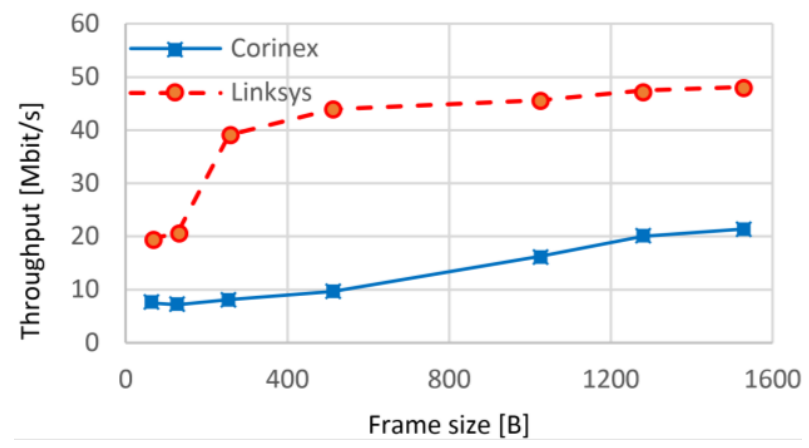

Fig. 13. Comparison of UDP throughput (symmetrical data flow) for Corinex and HomePlug AV modems for direct line of 50 meters' length.

Therefore, the comparison has to be made for all the proposed parameters (guidelines). The implementation of the proposed methodology therefore focuses on a detailed analysis of the results of all proposed complex parameters (guidelines), because from the point of view of application requirements, in PLC systems we are dealing with two contrary requirements: robustness or long range communication and high-speed data rates (throughput).

\section{DISCUSSION}

Chapter IV introduced a performance test for the "Modem-to-Modem" (point-to-point) connection for PLC communication according to the proposed methodology. Point-to-point PLC communications were tested in the experimental environment of isolated power line and included the typical noise impairments. This environment enables the same tests to be repeated under the same conditions for all PLC technologies.

The measurement set-up was considered only for point-topoint scenario, because the communication parameters for the whole PLC communication, e.g. in the case of repeaters, could be easily computed according to the communication parameters of particular paths (point-to-point between repeaters).

Building real Smart Metering networks is very expensive and time-consuming and, also, it is impossible to install different technologies in the same environment just for comparison. Therefore, an experimental laboratory environment with isolated power line and typical noise impairments is an easy, economical and time-affordable solution for a first comparison and evaluation of different PLC technologies and vendors.

Performance tests in a real Smart Metering network could be conducted according to the proposed methodology. In a real Smart Metering network environment, the methodology could also be extended by performance tests and measurements for communication between a data center (e.g. SCADA) and a Smart Meter with PLC module.

The methodology and performance tests were extended for communication between a data center and a Smart Meter with the following parameters:

- Evaluation of the time processing of a group of ad-hoc requests (on demand reading).

- Verification of relay response or switch disconnector response.

- Coverage vs. throughput.

- The time period of connection set-up.
- Availability in time according to the location.

- Establishing communication after a power outage.

- Impact of repeater outage on communication.

- Impact of repeaters on throughput.

- Impact of topology changes on communication.

Also other signicicant parameters are necessary to be evaluated, e.g. electromagnetic immunity or Power Spectral Density. Electromagnetic immunity tests were designed to conduct tests of modem immunity against voltage dips and short interruptions of the network voltage in accordance with EN 61000-4-11 standards. Power Spectral Density (PSD) ensures compliance with government regulations and ensures that no interference of Amateur Radio Services is generated in the high-frequency (HF) Amateur Radio bands.

The proposed methodology for the comparison and evaluation of PLC technology also considered the security issue, mechanical resistance, cost of installation and maintenance, standards and regulations issue, and so on.

\section{CONCLUSIONS}

For Smart Metering the best PLC technology must be identified and carefully selected. Not all PLC technologies are created equal and not all PLC technologies should be judged by the poor performance of some of them. In this article we have presented a methodology designed mainly for the performance evaluation of OFDM-based powerline communication systems.

The particular measurable parameters of the proposed methodology provide the guidelines for a first economical and time-affordable comparison and evaluation of different PLC technologies and PLC vendors but also for postdeployment performance tests.

The particular parameters of the proposed methodology could be used for different technologies but the aim of the proposed methodology was focused exclusively on the PLC, because PLC networks cannot be planned in the same way as other telecommunications technologies can.

\section{REFERENCES}

[1] S. Galli, A. Scaglione, Z. Wang, "For the grid and through the grid the role of power line communications in the smart grid", in Proc. IEEE, vol. 99, no. 6, 2011, pp. 998-1027. DOI: 10.1109/JPROC.2011.2109670.

[2] S. Chren, B. Rossi, T. Pitner, "Smart grids deployments within EU projects: The role of smart meters", Smart Cities Symposium Prague (SCSP 2016), Prague, 2016, pp. 1-5. DOI: 10.1109/SCSP.2016.7501033.

[3] P. Mlynek, J. Misurec, R. Fujdiak, Z. Kolka, L. Pospichal, "Heterogeneous networks for smart metering - power line and radio communication", Elektronika ir Elektrotechnika, vol. 21, no. 2, pp. 85-91, 2015. DOI: 10.5755/j01.eee.21.2.11515.

[4] Xi. Fang, Satyajayant Misra, Guoliang Xue, Dejun Yang, "Smart grid - the new and improved power grid: a survey", IEEE Communications Surveys \& Tutorials, vol. 14, no. 4, pp. 944-980, 2012. DOI: 10.1109/SURV.2011.101911.00087.

[5] A. Sendin, I. Pena, P. Angueira, "Strategies for power line communications smart metering network deployment", Energies, vol. 7, no. 4, pp. 2377-2420, 2014. DOI:10.3390/en7042377.

[6] Kyong-Hoe Kim, Han-Byul Lee, Yong-Hwan Lee, Seong-Cheol Kim, "PHY abstraction methodology for the performance evaluation of PLC channels", in Proc. IEEE Int. Symposium on Power Line Communications and Its Applications (ISPLC 2010), Rio de Janeiro, Brazil, 2010, pp. 28-32. DOI: 10.1109/ISPLC.2010.5479903.

[7] V. Oksman, S. Gallii, "G.hn: The new ITU-T home networking standard", IEEE Communications Magazine, vol. 47, no. 10, pp. 138-145, 2009. DOI: 10.1109/MCOM.2009.5273821. 
[8] S. Galli, M. Koch, H. Latchman, S. Lee, V. Oksman, "Industrial and international standards on PLC base networking technologies", Power Line Commun, New York: Wiley, 2010.

[9] P. Mlynek, M. Koutny, J. Misurec, Z. Kolka, "Measurements and evaluation of PLC modem with G3 and PRIME standards for street lighting control", in Proc. 18th IEEE Int. Symposium on Power Line Communications and its Applications (ISPLC 2014), Glasgow, Scotland, 2014, pp. 238-243. DOI: 10.1109/ISPLC.2014.6812318.

[10] A. Ikpehai, B. Adebisi, K. M. Rabie, "Broadband PLC for clustered advanced metering infrastructure (AMI) architecture", Energies, vol. 9 , no. 7, p. 569, 2016. DOI: 10.3390/en9070569.

[11] A. F. Pinto-Benel, M. Blanco-Velasco, F. Cruz-Roldan, "Throughput analysis for wavelet OFDM in broadband power line communications", Computer Science - Information Theory, vol. 03, 2017. [Online]. Available: arXiv: 1703.05100

[12] R. M. Oliveira, M. S. P. Facina, M. V. Ribeiro, A. B. Vieira, "Performance evaluation of in-home broadband PLC systems using a cooperative MAC protocol", Computer Networks, vol. 95, 2016 , pp. 62-76. DOI: 10.1016/j.comnet.2015.12.004.

[13] L. Jianming, Z. Bingzhen, G. Liang, Y. Zhou, W. Yirong, "Communication performance of broadband PLC technologies for smart grid", in Proc. IEEE Int. Symposium on Power Line Communications and Its Applications (ISPLC 2011), Udine, Italy, 2011, pp. 491-496. DOI: 10.1109/ISPLC.2011.5764448.

[14] A. M. Nyete, T. J. O. Afullo, I. Davidson, "Performance evaluation of an OFDM-based BPSK PLC system in an impulsive noise environment", in Proc. 35th PIERS Conf., Guangzhou, China, 2014, pp. 2010-2013.

[15] A. Mathur, M. R. Bhatnagar, B. K. Panigrahi, "Performance evaluation of PLC under the combined effect of background and impulsive noises", IEEE in Communications Letters, vol. 19, no. 7, pp. 1117-1120, 2015. DOI: 10.1109/LCOMM.2015.2429129.

[16] A. Mathur, M. R. Bhatnagar, B. K. Panigrahi, "Performance evaluation of PLC with log-normal channel gain over Nakagami-m additive background noise", in Proc. IEEE 26th Annual Int. Symposium on Personal, Indoor, and Mobile Radio Communication (PIMRC 2015), 2015, pp. 824-829. DOI 10.1109/PIMRC.2015.7343411.

[17] S. Mudriievskyi, R. Lehnert, "Performance evaluation of the G.hn PLC PHY layer", in Proc. 18th IEEE Int. Symposium on Power Line Communications and its Applications (ISPLC 2014), Glasgow, UK, 2014, pp. 296-300. DOI: 10.1109/ISPLC.2014.6812325.

[18] Y. Tsado, K. Gamage, D. Lund, "Performance of time-critical smart grid applications in narrow band power line communication", in Proc. 7th IET Int. Conf. Power Electronics, Machines and Drives (PEMD 2014), 2014, pp. 1-5. DOI: 10.1049/cp.2014.0458.

[19] V. N. Papilaya, A. J. H. Vinck, K. Ouahadaz, A. Mengi, M. Weinand, M. Koch, "Analysis of the devolo's $500 \mathrm{kHz}$ G3-PLC acces technology based on smart grid field trials", in Proc. 18th IEEE Int. Symposium on Power Line Communications and its Applications (ISPLC 2014), Glasgow, UK, 2014, pp. 138-143. DOI: 10.1109/ISPLC.2014.6812356.

[20] Echelon. The open smart grid protocol. [Online]. Available: www.echelon.com/technology/osgp.

[21] A. Maltsev, V. Sergeyev, A. Davydov Jr., A. Maltsev, E. Kalantzis, A. Valkanas, A., Alexiou, F. Boccardi, G. Paltenghi, K. Leung, O. Moreno, PHY abstraction methodology and SLS simulator implementation. IST-4-027310 Deliverable D5.1.2, 2007.

[22] J. Gilbert, W-J. Choi, Q. Sun, A. Tehrani, H. Ye, B. Jechoux, H. Bonneville, Unified Black Box PHY Abstraction Methodology. IEEE Contribution 802.11-04/0218r1, 2004.

[23] Ericsson. System level evaluation of OFDM-further considerations. TSGRAN WG1 \#35, R1-03-1303, 2003.

[24] K. Brueninghaus, D. Astely, T. Salzer, S. Visuri, A. Alexiou, S. Karger, G. A. Seraji, "Link performance models for system level simulations of broadband radio access systems", in Proc. IEEE 16th Int. Symposium on Personal, Indoor and Mobile Radio Communications (PIMRC 2005), Berlin, Germany, 2005, pp. 23062311. DOI: 10.1109/PIMRC.2005.1651855.

[25] R. Srinivasan, J. Zhuang, L. Jalloul, R. Novak, J. Park, IEEE 802.16m Evaluation Methodology document. IEEE Contribution $802.16 \mathrm{~m}-08 / 004 \mathrm{r} 4,2008$.

[26] A. Cataliotti, A. Daidone, G. Tine, "A medium voltage cable model for power line communication", IEEE Trans. Power Del., vol. 24 , no. 1 , pp. 129-135, 2009. DOI: 10.1109/TPWRD.2008.2002664.

[27] N. Zajc, N. Suljanovic, A. Mujcic, G. F. Tasic, "Frequency characteristics measurement of overhead high-voltage power-line in low radio-frequency range", IEEE Trans. Power Del., vol. 22, no. 4 , pp. 2142-2149, 2007. DOI: 10.1109/TPWRD.2007.905369.

[28] A. Cataliotti, V. Cosentino, D. Di Cara, G. Tine, "Simulation and laboratory experimental tests of a line to shield medium-voltage power-line communication system", IEEE Trans. Power Delivery, vol. 26, no. 4, pp. 2829-2836, 2011. DOI 10.1109/TPWRD.2011.2166813

[29] A. G. Lazaropoulos, P. G. Cottis, "Capacity of overhead medium voltage power line communication channels," IEEE Trans. Power Delivery, vol. 25 , no. 2, pp. 723-733, 2010. DOI: 10.1109/TPWRD.2009.2034907.

[30] A. G. Lazaropoulos, "Broadband transmission characteristics of overhead high-voltage power line communication channels", Progress In Electromagnetics Research B, vol. 36, pp. 373-398, 2012. DOI:10.2528/PIERB11091408.

[31] A. Cataliotti, A. Daidone, G. Tine, "Power line communication in medium voltage systems: characterization of MV cables", IEEE Trans. Power Delivery, vol. 23, no. 4, pp. 1896-1902, 2008. DOI: 10.1109/TPWRD.2008.919048.

[32] A. Cataliotti, P. Russotto, D. Di Cara, E. Telaretti, G. Tine, "New measurement procedure for load flow evaluation in medium voltage smart grids", in Proc. 2013 IEEE Int. Instrumentation and Measurement Technology Conf. (I2MTC 2013), Minneapolis, MN, 2013, pp. 517-522. DOI: 10.1109/I2MTC.2013.6555471.

[33] A. Cataliotti, D. Di Cara, R. Fiorelli, G. Tine, "Power-line communication in medium-voltage system: simulation model and onfield experimental tests", IEEE Trans. Power Delivery, vol. 27, no. 1, pp. 62-69, 2012. DOI: 10.1109/TPWRD.2011.2171009.

[34] A. Cataliotti, V. Cosentino, D. D. Cara, G. Tine, "Simulation of a power line communication system in medium and low voltage distribution networks", in Proc. IEEE AMPS Int. Workshop Appl. Meas. Power Syst., Aachen, Germany, 2011, pp. 107-111. DOI 10.1109/AMPS.2011.6090429.

[35] G. Artale, "Medium voltage smart grid: experimental analysis of secondary substation narrow band power line communication", IEEE Trans. Instrumentation and Measurement, vol. 62, no. 9, pp. 23912398, 2013. DOI: 10.1109/TIM.2013.2270924.

[36] A. Waadt, Ch. Kocks, G. H. Bruck, P. Jung, B. Sachsenhauser, "Achievable data rates of broadband power line communications in an underground medium-voltage network", Journal of Green Engineering, vol. 3, pp. 245-259, 2013.

[37] O. Vondrous, P. Macejko, T. Hegr, Z. Kocur, "Testing methodology for performance evaluation of communication systems for Smart Grid", in 2nd Int. Conf. Intelligent Green Building and Smart Grid (IGBSG 2016), Prague, 2016, pp. 1-6. DOI 10.1109/IGBSG.2016.7539412.

[38] A. Sendin, I. Berganza, A. Arzuaga, X. Osorio, I. Urrutia, P. Angueira, "Enhanced operation of electricity distribution grids through smart metering plc network monitoring, analysis and grid conditioning", Energies, vol. 6, pp. 539-556, 2013. DOI 10.3390/en6010539.

[39] Corinex. LV High Density Compact Access Gateway CXP-HDCGWYC. [Online]. Available: http://www.corinex.com/ series/gateways- 0 .

[40] Corinex. Medium Voltage Compact Gateway CXP-HDCV2-GWYC [Online]. Available: http://www.corinex.com/product/mediumvoltage-compact-gateway.

[41] P. Mlynek, J. Misurec, M. Koutny, P. Silhavy, "Two-port network transfer function for power line topology modelling", Radioengineering, vol. 21, no. 1, pp. 356-363, 2012.

[42] J. Hrad, J. Vodrazka, "Modelling of data communication over medium-voltage power distribution lines", Communications, vol. 18, pp. 62-66, 2016

[43] J. Vodrazka, J. Hrad, "Communication over middle-voltage distribution lines with inter-section crosstalk modeling", in 17th Int. Conf. Mechatronics - Mechatronika (ME 2016), Prague, Czech Republic, 2016, pp. 533-536.

[44] P. Mlynek, Z. Hasirci, J. Misurec, R. Fujdiak, "Analysis of channel transfer functions in power line communication system for smart metering and home area network", Advances in Electrical and Computer Engineering, vol. 16, pp. 51-56, 2016. DOI: 10.4316/AECE.2016.04008.

[45] Z. Hasirci, M. Ozturk, I. H. Cavdar, R. Benveniste, H. Kaya, "Investigating the effects of line length \& branch number on busbar broadband PLC systems", in Proc. Int. Conf. Telecommunications and Signal Processing (TSP 2017), Barcelona, 2017, pp. 247-250. DOI: 10.1109/TSP.2017.8075979. 